\title{
Science, vérité et croyances
}

\section{Andreas Bikfalvi}

$>$ Cet article se propose de discuter certains aspects de la relation entre la science, la vérité et les croyances, en se concentrant principalement sur l'activité scientifique des sciences biologiques et médicales, et sur la façon dont elle se rapporte aux notions de vérité et de croyance. La relation avec les religions ne sera donc pas abordée. La science utilise des méthodes spécifiques pour acquérir le savoir, et les philosophes ont analysé comment cette connaissance scientifique était acquise, en tentant d'en préciser les démarches et d'établir quelques règles générales. Certains théoriciens radicaux ont ainsi contesté la valeur de la méthode scientifique, malgré le succès indiscutable de la science. Si elle est rationnelle, la science n'est cependant pas exempte de croyance, ce qui peut avoir un impact positif ou négatif sur l'acquisition des connaissances scientifiques et sur l'idée de la nature humaine. <

Bien avant la révolution scientifique, il y a 500 ans, science et croyances avaient des rôles majeurs dans nos sociétés. Les systèmes de croyances sont apparus à un moment où l'humanité n'avait pas encore les outils conceptuels pour expliquer des phénomènes naturels, comme le mouvement du soleil, de la lune, les éclairs, le tonnerre, ou l'apparitions de la vie. L'homme, en cherchant des explications, s'est réfugié dans ce qui était le plus immédiat pour lui, la croyance et le surnaturel. Le biologiste et éthologiste britannique Richard Dawkins voyait dans la croyance au surnaturel un virus mental [1]. D'autres, comme le sociologue français Émile Durkheim, voyaient dans la croyance, notamment religieuse, un élément structurant de la société agissant comme facteur cohésif [2].

L'une des formes les plus organisées des systèmes de croyance est représentée par la religion, qui avait une influence importante sur la science. Le théologien et médecin français d'origine espagnole Michel Servet illustre

Vignette (Photo () Inserm).

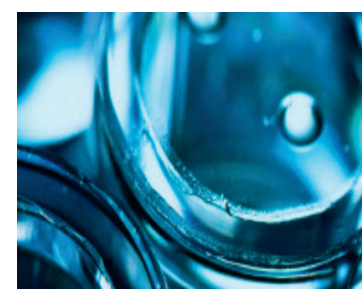

Laboratoire de l'angiogenèse et du microenvironnement des cancer, Inserm U1029, Université de Bordeaux, Allée Geoffroy St-Hilaire, 33615 Pessac, France, et Institut d'Histoire et Philosophie des Sciences et Techniques (IHPST), 13, rue du Four, 75006 Paris, France. andreas.bikfalvi@u-bordeaux.fr

très bien ce point. II publia en effet un livre théologique intitulé « Cristianissimi Restitutio » dans lequel il discuta de la trinité et de la personne de Jésus [3]. Dans un passage de ce livre, il tenta d'expliquer comment l'esprit vital pénétrait le corps, et y développa l'idée de la circulation pulmonaire pour expliquer ce phénomène. Ce type de mélange entre théologie et science ne se vît plus guère ultérieurement, et le médecin William Harvey, père des lois de la circulation sanguine, n'y fait plus référence dans « de moto cordis in sanguinis animalibus », publié en 1628 [4]. La science s'est donc progressivement détachée de l'influence des religions. Cependant, elle n'a pu se détacher complétement des croyances non religieuses comme nous allons le développer ci-dessous.

Notre article va en effet aborder certains aspects de la relation entre science, vérité et croyance(s). Vérité et croyance(s) ne seront pas abordées dans leur contexte religieux (ce qui relève de la foi). Nous discuterons essentiellement des impacts positif et négatif des croyances sur l'acquisition des connaissances scientifiques et de l'idée de la nature humaine. Une critique du relativisme dans les sciences et ses répercussions sur la sphère sociétale sera également formulée.

\section{La nature de l'activité scientifique}

Dans ce chapitre, ne seront abordées que les sciences naturelles, en particulier les sciences biologiques et médicales, pour lesquelles l'activité scientifique est majoritairement liée à l'expérimentation.

\section{Méthodes utilisées en sciences biologiques et médicales ${ }^{1}$}

Dans les sciences biologiques et médicales, l'activité scientifique opère dans sa pratique, pour l'essentiel, selon deux modes : l'inférence inductive et l'inférence déductive. L'inférence inductive repose sur l'analyse des données collectées systématiquement dans un dispositif expérimental, données servant ensuite à I 'élaboration d'hypothèses et de concepts qui, à leur tour, pourront être testés. À l'opposé, l'infé-

${ }^{1}$ Les paragraphes concernant la méthodologie scientifique sont extraits du livre de l'auteur « Une brève histoire du vaisseau sanguin et lymphatique » publié par EDP Science en 2016. 
rence déductive formule des hypothèses qui, ensuite, sont mises à l'épreuve par l'expérimentation.

L'inférence causale est la méthode la plus importante de l'inférence inductive dans le cadre de l'expérimentation. Elle établit un lien causal entre les faits qui sont observés dans un dispositif expérimental et utilise la méthode des différences. On trouve ainsi dans les écrits du philosophe logicien John Stuart Mill [5] une des premières description de la méthode des différences. II y écrit ${ }^{2}$ : «Si à un moment un événement a lieu et à un autre moment il n'a pas lieu et toutes les conditions sont en commun à l'exception d'une seule (qui existe seulement dans le premier événement), la condition qui est différente entre ces deux événements est la cause ou une partie indispensable de la cause du phénomène observé ». On peut exprimer cela de manière simplifiée, en disant que si le comportement d'un système expérimental varie en fonction d'une seule condition, alors cette variation est due, toute ou en partie, à cette dernière condition. Ainsi, si nous pouvons identifier un facteur, et seulement un, qui diffère entre deux situations/conditions, alors ce facteur a une importance causale pour l'événement.

L'inférence causale nécessite néanmoins d'être renforcée par des conditions et/ou critères additionnels. Les deux situations comparées doivent être homogènes, et l'agent ou le mécanisme potentiellement causal de la réponse induite doit n'être présent que dans l'une des situations et absent dans l'autre. D'autres facteurs qui pourraient être impliqués ne doivent pas être présents au moment de l'expérience. L'inférence causale est en fait un moteur essentiel de la stratégie de découverte en biologie et en médecine. Elle permet d'extrapoler, par exemple, une connexion causale potentielle entre une altération moléculaire et un phénotype observé. Elle nécessite d'être renforcée par d'autres approches expérimentales, comme nous le verrons plus loin. L'inférence déductive à partir d'hypothèses est, quant à elle, une stratégie répandue dans d'autres sciences que la biomédecine, dans lesquelles une théorie générale existe et à partir de laquelle des hypothèses peuvent être déduites, comme en physique par exemple. En biologie, il n'existe pas à proprement parler de théories similaires à celle de la relativité ou de la mécanique quantique. En revanche, il existe un ensemble d'observations, de constats et d'affirmations à partir desquels des hypothèses peuvent être déduites.

Les inférences inductive et déductive sont intimement liées. L'inférence inductive génère des hypothèses qui ensuite peuvent être testées dans une démarche déductive. On est ainsi dans une relation «œuf-poule », dont on peut se demander qui en est le moteur initial. Les déductivistes, dans la lignée du philosophe des sciences Karl Popper [6], pensent que le « déductivisme » prime par rapport à l'induction, car notre démarche cognitive nécessite des présuppositions afin de pouvoir analyser les données qui sont générées. Dans la démarche déductiviste poppérienne, le critère de falsification est essentiel dans l'acquis du savoir. Par ce critère, une hypothèse est plus proche de la «vérité » si celle-ci a le plus de chance d'être falsifiée mais qu'elle

\footnotetext{
${ }^{2}$ «If an instance in which the phenomenon under investigation occurs, and an instance in which it does not occur, have every circumstance in common save one, that one occurring only in the former; the circumstance in which alone the two instances differ, is the effect, or the cause, or an indispensable part of
} the cause, of the phenomenon ». ne l'est pas. Nous verrons plus loin la limite de cette démarche.

L'approche bayésienne est une approche statistique qui assigne une probabilité à un événement en relation avec une autre condition [7]. D'une manière générale, la méthode développée par le mathématicien Thomas Bayes au XVIII ${ }^{e}$ siècle, postule que la probabilité d'un événement associée à une condition « $X$ » est la résultante de la probabilité de « $X »$ multipliée par la probabilité que l'événement qui lui est associé ait lieu, divisé par la probabilité que l'événement apparaisse seul. L'approche Bayésienne, revenue à la mode depuis 1990, a trouvé un certain intérêt en biologie (notamment dans l'analyse des données de génomique et la modélisation), en épidémiologie et dans la recherche clinique et les essais thérapeutiques.

Une autre approche est celle de l'erreur statistique (error statistical approach) [8]. II n'y a, dans cette approche, pas de probabilité attribuée à des hypothèses, mais la chance avec laquelle une hypothèse va passer les tests avec succès est évaluée. Plus précisément, on s'interroge sur la chance qu'a une hypothèse nulle (null-hypothesis), c'est-à-dire que l'événement n'existe pas, de passer le test avec succès: si la probabilité de l'hypothèse nulle est élevée, alors cela peut signifier que l'observation n'est pas due au lien causal considéré. Deux erreurs peuvent être faites dans ce cas : le rejet de l'hypothèse nulle, qui entraîne une certaine probabilité d'accepter une hypothèse alternative fausse (erreur de type I) ; le rejet de l'hypothèse alternative, qui entraîne une certaine probabilité d'accepter une hypothèse nulle pourtant fausse (erreur de type 2). Cette approche a donc de fortes similitudes avec le critère de falsification, pour lequel une théorie semble la plus solide si elle a un risque important d'être falsifiée mais qu'au bout du compte elle ne l'est pas [6].

L'approche d'inférence de la meilleure explication (inference to the best explanation, ou IBE) est intéressante en biologie [9]. Aussi appelée abduction, cette approche comble le vide entre l'inférence inductive, exclusivement fondée sur le lien causal, et les explications mécanistiques, qui reposent sur des notions comme la topologie, la structure, le rétrocontrôle etc. ${ }^{3}$ Un élément intéressant à introduire ici est la notion de «réseau d'évidences cumulatives», appelé réseau nomologique d'évidences cumulatives par certains, et qui a pour origine le domaine de la psychologie [11]. Des évidences issues de recherches réalisées avec différentes méthodes et approches doivent converger

\footnotetext{
${ }^{3}$ Pour des informations détaillées sur ces différentes approches, le lecteur pourra
} consulter les écrits de Marcel Weber [10]. 
pour valider une hypothèse ou une théorie. Cette notion a une utilité dans les sciences biologiques et médicales. Il est cependant important que les évidences soient générées par des méthodes et approches indépendantes, multiples et diversifiées, et les données provenant de l'expérimentation peuvent être complétées par des données observationnelles, qui peuvent être intégrées dans le réseau d'évidences cumulatives.

\section{Facteurs influençant la démarche scientifique}

Pour la plupart des chercheurs, l'ultime but est d'obtenir des connaissances sur la façon dont le monde (la nature, les faits de sociétés, etc.) est structuré, et d'en déduire les instruments/procédés qui ont une utilité pour l'évolution de ce monde (prédictions, technologies, etc.). Une façon simpliste (ou ultra-réaliste) de formuler cela serait: « connaître le monde tel qu'il est ». Ceci suppose une conception du monde pour laquelle les méthodes mises en œuvre pour l'acquisition des connaissances et l'appareil cognitif de l'investigateur n'auraient pas de rôle décisif sur la qualité de la connaissance acquise. Pour beaucoup de scientifiques, ce présupposé est implicite. Une autre acception, diamétralement opposée (on pourrait dire idéaliste), serait de dire que notre connaissance du monde dépend, pour l'essentiel, de notre appareil cognitif. La réalité, telle qu'elle est, n'est cependant jamais sondable. Notre connaissance du monde dépend en fait de notre perception, reposant sur nos organes sensoriels et notre capacité cérébrale à décoder et à structurer les informations recueillies. Nos organes des sens, principalement visuel et auditif, présentent certaines limitations de perception. On peut donc dire que le monde tel que nous le percevons dépend des possibilités et des limites de nos organes sensoriels. Notre monde est donc un «monde moyen » limité par des frontières de perception et de cognition.

Ces deux conceptions souffrent, toutes deux, de graves défauts. La conception ultra-réaliste a des limites sur le plan scientifique pour la connaissance de l'infiniment petit. En physique quantique, les limitations de nos fonctions de perception nous ont conduits à mettre en place des équations mathématiques probabilistes n'ayant pas l'ambition de décrire la réalité telle qu'elle est, mais de doter le scientifique d'une capacité prédictive du comportement de l'infiniment petit. Au contraire, la conception idéaliste présente le danger du subjectivisme et du relativisme.

Un autre aspect à prendre en considération est l'influence du langage dans l'acquisition des connaissances dans les sciences. Ce langage peut être mathématique pour certaines d'entre elles. Pour une (grande) partie des sciences (notamment les sciences du vivant), il est surtout nominal. On nomme les choses et étiquette, pour ainsidire, une entité ou un fait. Les objets ainsi nommés sont ensuite mis en relation. On peut alors se demander si l'utilisation de notre langage influence ce que nous voulons connaître, et orienter la façon par laquelle nous pratiquons la science.

Afin d'illustrer cela, prenons un exemple issu de la recherche en biologie vasculaire, un domaine de recherche que je connais bien. Le vascular endothelial growth factor (VEGF) a été découvert par Napoléon Fer- rara et Jean Plouet, en 1989, pour sa capacité à stimuler exclusivement les cellules endothéliales vasculaires [11]. On pensait alors à l'époque qu'il s'agissait d'un facteur exclusivement vasculaire. L'absence d'un seul des deux allèles conduisait chez la souris à un phénotype vasculaire (pas de complémentation par le second allèle). L'inactivation du gène codant le VEGF était donc associée à une haplo-insuffisance. Par la suite, il fut montré que les cellules endothéliales n'étaient pas les seules cellules à exprimer des récepteurs du VEGF : les neurones, les cellules de Leydig et certaines cellules tumorales en possèdaient également.

On peut donc se demander comment la recherche sur ce facteur aurait été orientée si ses propriétés avaient été initialement découvertes non dans le système vasculaire, mais dans le système nerveux? II aurait pu être alors appelé « Neurotrophine- $X$ » et ceci aurait eu un impact sur les programmes de recherches établis par les différentes agences de recherches nationales et internationales. On peut donc penser que l'orientation de la recherche aurait été très différente si ce facteur avait été découvert par les neuroscientifiques et non par les biologistes vasculaires.

Je ne dis pas que le fait de nommer va significativement altérer ce que nous recherchons, je dis seulement que l'orientation des recherches peut être influencée par la manière dont nous étiquetons un objet.

Un autre aspect de l'influence de notre cognition et du langage sur ce que nous recherchons est représenté par les schémas et diagrammes que nous dessinons pour nous faire une idée des liens causaux et séquentiels dans une chaîne d'événements biologiques et de comprendre leurs imbrications parfois complexes. Si nous prenons, par exemple, la signalisation cellulaire, nous dessinons nos schémas en fonction des connaissances que nous avons à l'époque précise où nous réalisons nos recherches. À l'heure actuelle, les voies de signalisation cellulaire reposent sur différents types de récepteurs, décrits selon leur structure et leur mécanisme d'activation : les récepteurs de type tyrosine kinase, les récepteurs de type sérine/thréonine kinase, les récepteurs couplés aux protéines $G$, etc. Ces récepteurs sont couplés, grâce à différentes molécules adaptatrices, à des cascades de signalisation intracellulaire qui, ultimement, activeront dans le noyau de la cellule les facteurs de transcription d'un ensemble de gènes contrôlant finement le phénotype cellulaire. La nature chimique de ces récepteurs et de ces molécules de signalisation ne sera probablement pas fondamentalement révisée par les découvertes futures, mais le détail du réseau de signalisation cellulaire sera probablement très affiné dans les prochains 50 ans. 
Mettons ainsi en lien, avec ce propos sur le langage, les notions de «mécanisme » et de «pathway». Ces deux notions sont-elles, en fait, interchangeables? Certains philosophes des sciences pensent que non [12]. Pour eux, il s'agit de concepts fort différents. La notion de «mécanisme » donne une explication détaillée d'une relation causale hautement résolutive. On peut ainsi penser au mécanisme d'activation de certaines enzymes et à leur interaction avec les coenzymes, dont la description repose sur des études sophistiquées utilisant des méthodes de biochimie et de biologie structurale, comme la RMN (résonnance magnétique nucléaire) ou la cristallographie. Un «pathway » est, lui, constitué d'une séquence d'entités moléculaires connectées entre elles, qui conduisent à la modulation d'une fonction donnée (prolifération cellulaire, migration cellulaire, survie, etc.), comme pour les voies de signalisation qui sont initiées par des récepteurs de type tyrosine kinase ou des voies métaboliques. La nature structurale détaillée, «chimique », de la connectivité peut dans ce cas ne pas être de première importance pour l'élaboration d'un concept. II suffit de mettre en évidence une connectivité et de la caractériser comme activatrice ou inhibitrice d'une fonction. Un «pathway » est ainsi composé de différents modules moléculaires qui sont connectés entre eux par des mécanismes fins (ces connections pouvant être, par ailleurs, structuralement renseignées de manière très détaillée).

II s'agit donc, entre «pathway » et «mécanisme », d'une différence de résolution ou de niveau d'analyse : le niveau «macroscopique» étant représenté par le «pathway », et le niveau «microscopique », par le mécanisme. Les scientifiques ne font que rarement cette distinction et utilisent ces notions indifféremment. Ainsi l'élucidation d'un «pathway » peut devenir compréhension d'un mécanisme, comme, par exemple, la mise en évidence et la validation d'une voie de signalisation décrite comme causale pour la migration cellulaire. On peut donc se demander de l'utilité de cette distinction conceptuelle pour la pratique scientifique.

On pourrait penser que je privilégie la conception idéaliste, qui postule que l'acquisition du savoir est principalement le reflet de notre appareil cognitif. Ceci n'est pas le cas. L'appareil cognitif se forme au contact de la réalité. Il y est imbriqué selon un cercle d'apprentissage vertueux. L'observation du développement des facultés cognitives chez l'enfant illustre très bien ce processus.

La réalité et les lois intrinsèques de la nature peuvent être appréhendées par l'esprit humain. L'homme est même capable de développer des outils et des interfaces (ordinateurs, programmes de calcul, appareillage expérimental, outils moléculaires, etc.) qui assistent ses mécanismes cognitifs pour l'exploration du monde. Ces outils et interfaces sont en quelques sorte des facultés cérébrales externalisées et matérialisées qui obéissent à des règles inhérentes de l'esprit humain (règles logiques, vérification, mise à l'épreuve, etc.). Ces outils et interfaces deviendront certainement de plus en plus complexes et modifieront notre façon de cartographier la réalité. Cette vision du monde est la plus compatible avec la réalité et le développement scientifique et technologique.

\section{La nature des croyances}

Eléments de « croyance » dans l'activité scientifique La croyance est un élément essentiel de nos sociétés humaines. Nous croyons que la terre est ronde, sans jamais avoir fait un voyage spatial pour le voir de nos propres yeux... Nous croyons que les cartes du monde sont correctes et nous permettent de voyager d'un endroit à un autre sur la planète. Nous croyons avec une confiance mesurée les prévisions météorologiques... Nous croyons à l'existence de l'évolution biologique. Nous croyons que le monde est fait d'atomes et d'éléments chimiques et obéit aux lois fondamentales de la physique... Nous croyons le médecin quand il fait un diagnostic et nous prescrit un traitement...

Qu'ont ces croyances en commun? Elles sont toutes fondées, pour l'essentiel, sur des évidences directes ou indirectes et ont une explication rationnelle (croyance de type 1) (Figure 1). Les évidences indirectes reposent sur des connaissances acquises par autrui dont le statut, la méthode etc. sont crédibles, car testables par l'expérience. Certaines d'entre elles, mais pas toutes, peuvent aussi être reproduites. Ce type de croyance est tout à fait compatible avec la science. Il est même indispensable à l'activité scientifique. Si nous ne croyons pas aux connaissances acquises par nos ainés, comment pouvons-nous bâtir de nouvelles connaissances et faire en sorte que le savoir progresse? C'est tout à fait indispensable au progrès de notre savoir. II existe des situations dans lesquelles ces croyances nécessitent d'être mises à l'épreuve et questionnées. Ces questionnements peuvent alors aboutir à la mise en question radicale d'une théorie complète, ou d'un système de croyance en vogue à un certain moment de l'évolution de nos connaissances. La mise à l'épreuve doit être, dans ce cas, fondée sur des évidences extrêmement solides. Elle est principalement associée à des révolutions scientifiques. La découverte du système circulatoire par W. Harvey est un exemple lumineux de cette remise en question [4]. S'il avait persisté et poursuivi la doctrine galénique ${ }^{4}$, il n'aurait pu découvrir les lois de la circulation sanguine. Harvey a eu l'obligation d'envisager un changement de paradigme pour constituer un nouveau cadre conceptuel dans lequel les recherches futures puissent évoluer. Ce changement a eu lieu grâce à sa confiance dans la méthode expérimentale et à l'analyse logique des observations obtenues, et non à la croyance dans les théories anciennes.

\footnotetext{
${ }^{4}$ Décrite par Galien.
} 


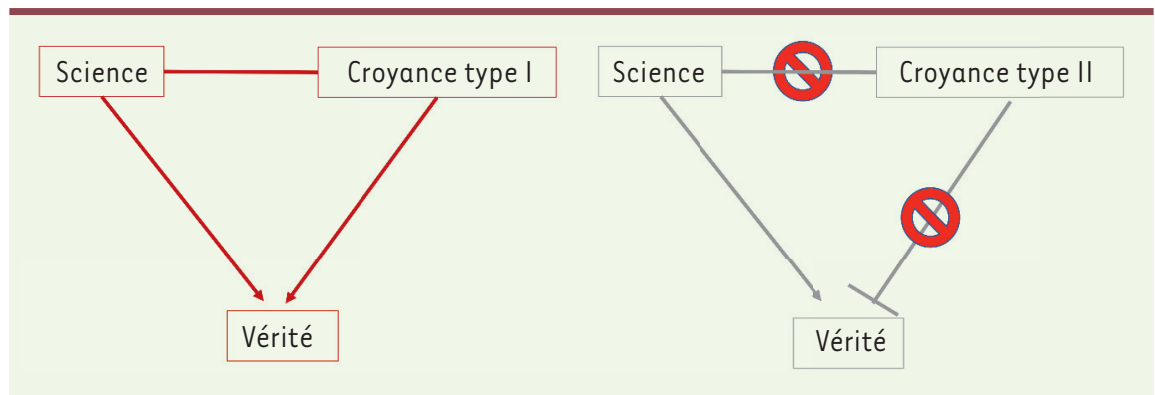

Figure 1. La figure de gauche montre la relation entre science, croyance et vérité dans le cadre d'une croyance de type I (croyance fondée sur un savoir antérieur et des évidences directes ou indirectes). Dans ce cas, la croyance a un effet positif. La figure de droite montre l'effet de la croyance de type II (croyance fondée sur l'héritage culturel). Dans ce cas, l'effet sur la connaissance et la vérité peut être nul voir négatif. Un bon exemple pour la croyance de type II est l'affaire Lyssenko (voir le texte).

\section{Croyances et mythes}

Les croyances que nous venons de décrire sont différentes de celles fondées sur l'héritage culturel (croyance de type II) (Figure I) qui sont liées aux mythes accumulés par l'humanité durant des siècles et jusqu'à une époque récente. Ces mythes ont tous plus au moins un caractère « religieux ». Ils sont en particulier retrouvés dans des systèmes idéologiques, dont l'effet peut devenir toxique. Un exemple de cet effet toxique est représenté par l'affaire Lyssenko qui, croyant au dogme communiste, a rejeté le darwinisme et la génétique, empêchant le développement de la génétique et de la biologie moléculaire en Union soviétique [13].

L'humanité a été une grande génératrice de mythes et leur apparition est probablement concomitante de l'évolution cognitive des Homo sapiens, il y a environ 40000-70000 ans. L'une des fonctions de ces mythes est sociale. Ils peuvent donner un sens à la vie de ceux qui y croient. Mais les mythes peuvent-ils nous révéler une sorte de «vérité » et, si oui, de quelle « vérité » peut-il s'agir?

Le philosophe allemand Kurt Hübner, dans son livre intitulé « die Wahrheit de Mythos » (La Vérité du Mythe) [14], posait le postulat que la vérité mythologique est toujours active aujourd'hui, et qu'elle représenterait une vision du monde équivalente à celle de la science. Les mythes se réfèrent à des histoires qui reflètent des angoisses existentielles qui se sont accumulées au cours de l'histoire de l'humanité. Ce sont, en quelque sorte, des histoires qui peuvent révéler des archétypes psychologiques profonds, comme le persona, le père, la mère, le héros, le dragon, etc., selon Carl Gustav Jung [15]. Ces mêmes archétypes peuvent se retrouver dans les contes, comme ceux des frères Grimm. Mais s'agit-t-il pour autant de vérités? Un mythe ne réclame pas de vérité factuelle : il se réfère souvent à des entités dont l'existence n'est qu'hypothétique. Une « vérité » morale peut néanmoins exister. Certains ont appelé cette vérité, «vérité métaphorique » [16]. II s'agit dans ce cas plutôt de signification ou de valeur, qui sont clairement à distinguer des vérités factuelles qui, elles, peuvent être mises à l'épreuve.

\section{Biais cognitifs}

Le biais cognitif s'apparente à la croyance. II peut avoir des effets positifs et négatifs. Dans son livre «Thinking, Fast and Slow » [17], le psychologue américain Daniel Kahneman définit deux façons de penser: l'une rapide, faisant appel à des automatismes cognitifs; l'autre, lente, représentée essentiellement par la pensée rationnelle. La première peut comporter des biais cognitifs de différents types comme, par exemple, l'heuristique de disponibilité ${ }^{5}$ (availability heuristics), le biais négatif (negative bias), la substitution d'attributs (attribute substitution), le biais optimiste (pervasive optimistic bias), l'effet de cadrage (framing effect), le biais de confirmation (confirmation bias), etc. Ces biais peuvent influencer positivement ou négativement la démarche scientifique. Le biais de confirmation, par exemple, peut pousser un chercheur à orienter ses expériences de manière à confirmer sa théorie ou ses résultats antérieurs, et ainsi l'éloigner de la vérité. Un exemple en recherche médicale est donné par les recherches portant sur le rôle des progéniteurs de cellules endothéliales dans le développement des cancers. Il a en effet été postulé que ces cellules contribuaient d'une manière décisive au développement de la vascularisation tumorale et pourraient constituer une cible thérapeutique majeure [18]. Ceci fut étayé par de nombreuses publications. II a suffi néanmoins d'une publication critique, dans la revue Proc Natl Acad Sci USA, qui contredisait cette affirmation, pour discréditer tout ce domaine de recherche [19]. Cela fait penser au petit garçon dans le conte d'Hans Christian Andersen «Les habits neufs de l'empereur» qui disait «Mais il n'a pas d'habit du tout $»^{6}$. Par la vertu d'une seule phrase, le constat objectif d'un enfant terrasse la vision subjective partagée par toute une communauté.

\section{Raison, science, vérité et croyance}

Raison, science et croyance sont intimement liées à la notion de vérité. Est-ce que la vérité est une notion

\footnotetext{
${ }^{5}$ Désigne la tendance ou le mode de raisonnement des personnes qui, sur une question donnée, privilégient et surestiment les informations immédiatement disponibles à notre mémoire. Les personnes s'abstiennent de rechercher de nouvelles informations qui pourraient éclairer la question.

${ }^{6}$ [...] C'est ainsi que l'Empereur marchait devant la procession sous le magnifique dais, et tous ceux qui se trouvaient dans la rue ou à leur fenêtre disaient: "Les habits neufs de l'empereur sont admirables ! [...]» Personne ne voulait laisser paraître qu'il ne voyait rien, puisque cela aurait montré qu'il était incapable dans sa fonction ou simplement un sot. Aucun habit neuf de l'empereur n'avait connu un tel succès. «Mais il n'a pas d'habit du tout! », dit un petit enfant dans la foule. [...] Et le cortège poursuivit sa route et les chambellans continuèrent de porter la traîne, qui n'existait pas.
} 
objective, immuable et déconnectée de notre appareil de cognition ? Cette position est globalement ultra-réaliste. Est-ce que la vérité est subjective, inhérente à notre appareil de cognition et conditionnée par ce dernier? Cette position est idéaliste et peut aboutir au relativisme.

\section{La tradition philosophique critique et la science}

La tradition philosophique critique, initiée par les philosophes écossais David Hume et allemand Emmanuel Kant, s'est propagée au $\mathrm{Xx}$ siècle avec l'empirisme logique du cercle de Vienne (avec Rudolf Carnap, Otto Neurath, etc.) [20, 21], puis avec Karl Popper, Imre Lakatos, Thomas S Kuhn et Paul Feyerabend [22-25]. Ces courants de pensée se distinguent par l'analyse philosophique des fondements des sciences et la solidité des méthodes utilisées. Si Rudolf Carnap élaborait une logique inductive pour servir de méthodologie générale dans les sciences, Karl Popper faisait de la méthode déductive la reine de la science, et introduisait la falsification comme critère de démarcation. Héritiers de K Popper, TS Kuhn et I Lakatos ont fait une analyse critique de la pensée de leur maître. Kuhn voyait dans le progrès scientifique non pas une accumulation des connaissances mais un avancement en dents de scie, et introduisit la notion de science normale, de paradigme et de révolution scientifique [26]. La science normale constitue l'activité du scientifique qui remplit un cadre exploratoire prédéfini par un paradigme préexistant. À un moment donné, des anomalies qui ne cadrent plus avec la théorie existante s'accumulent et la science entre en crise: une révolution scientifique apparaît. Elle permet l'élaboration d'un nouveau paradigme dans lequel la science normale pourra évoluer. Le concept de Kuhn est relativiste: il ne hiérarchise pas la connaissance accumulée à une époque par rapport à une autre. La valeur explicative est égale pour toutes les connaissances/savoirs et correspond à une évolution de la science à une certaine époque. Les exemples que Kuhn donne pour étayer sa théorie proviennent principalement de la physique. Mais est-ce vraiment correct? Prenons des exemples dans un domaine dont Kuhn n'était pas familier, la biologie, et en particulier le système circulatoire. Pouvons-nous vraiment dire que la théorie Galénique est équivalente à la théorie circulatoire de Harvey? Assurément non, car la théorie Galénique a été démantelée par l'expérimentation et la théorie circulatoire a alors constitué le fondement de toute la biologie cardiovasculaire et la physiologie du vivant. On ne peut même plus parler de théorie dans le sens où celle-ci ne peut plus être falsifiée. II ne s'agit donc pas vraiment d'une théorie mais d'un socle dont les théories/hypothèses qui en dérivent peuvent être testées et mises à l'épreuve (STHD). Pouvons-nous douter que la théorie circulatoire sera mise un jour en échec? Comment pouvonsnous reconnaître quand une théorie devient socle STHD?

Posons-nous la question. Avec quelle probabilité pouvons-nous affirmer que le système sanguin des vertébrés n'est pas circulatoire? Quelles sont les chances qu'un jour, un organisme vertébré possède un système sanguin ouvert dans lequel le sang ne re-circule pas? Cette probabilité est extrêmement faible, voire nulle, car tous les vertébrés examinés à ce jour possèdent un système sanguin circulatoire.

Nous parlerons donc de STHD lorsqu'une théorie a une probabilité quasi nulle d'être réfutée et ceci dans un future proche ou lointain.
Cette probabilité est mesurée par l'examen passé de toutes les occurrences d'une propriété $f(x)$ affectant l'objet $x$. Comme indiqué plus haut, les STHD peuvent contenir des sous-théories et hypothèses. Prenons, encore une fois, le système circulatoire comme exemple. La démonstration du système circulatoire des organismes vertébrés conduit tout droit à diverses sous-théories et hypothèses comme le postulat d'un système cellulaire qui relie la circulation veineuse et artérielle, ou les mécanismes impliqués dans le transport d'oxygène, d'éléments nutritifs ou de cellules vers les tissus, etc. Concernant le transport d'éléments provenant de la circulation sanguine vers les tissus, deux théories ont été formulées au XIX siècle: la théorie «corpusculaire » et la théorie «humorale » [27]. Le physiologiste anglais John Hughes Bennet avait formulé la théorie humorale dans laquelle seule la partie du sang non cellulaire avait un rôle à jouer et pouvait être transporté à travers la paroi vasculaire. À l'opposé, le médecin anglais William Addison avait émis une théorie corpusculaire dans laquelle les éléments solides (corpusculaires/cellulaires) pouvaient être transportés à travers la paroi vasculaire. Ces théories furent testées et l'immunologiste Julius Cohnheim montra qu'en effet des cellules pouvaient traverser la paroi.

\section{Critique du relativisme}

Paul Feyerabend dans son livre «Contre la méthode» et dans d'autres écrits va plus loin que Kuhn [28]. Pour lui, il n'y a pas de méthode pour faire la science, et il utilise l'expression «anything goes » pour dire que tous les systèmes d'acquisition de connaissances sont équivalents. II était un penseur complexe et sa critique était principalement dirigée contre ces philosophes des sciences qui essaient de définir des règles de conduite de l'activité scientifique [29]. Même si Feyerabend était critique vis-à-vis des rationalistes et des relativistes [29], son anarchisme philosophique, généralisé à l'ensemble de l'activité scientifique rend sa position extrêmement dangereuse car elle ne permet plus de distinguer le vrai du faux, ce qui conduit au relativisme. Au-delà des sciences, l'élargissement de ce concept à la sphère sociétale peut conduire au règlement des différences par des relations de pouvoir plutôt que par l'échange rationnel d'idées en quête d'un consensus. Le philosophe mathématicien anglais Bertrand Russell voyait dans cette position une justification du fascisme [30]. Pour lui, les doctrines des irrationalistes modernes privilégiaient la volonté plus que la pensée et le sentiment, l'intuition pour la formulation des propositions plus que l'observation et le raisonne- 
ment inductif, et la glorification du pouvoir et de la puissance ${ }^{7}$. Le constructivisme et le relativisme du sociologue des sciences Bruno Latour est à rapprocher de ce qui vient être dit. Dans son article publié dans La Recherche à propos de Ramsès II et du bacille de Koch [31], il maintient que Ramsès II n'aurait pas pu mourir de la tuberculose car avant sa découverte par Robert Koch le bacille n'avait pas d'existence réelle! II y a donc, ici, confusion entre identité et concept. $\varepsilon t$, de manière similaire, pourquoi ne pas dire que l'évolution des espèces n'a débuté que lorsque le concept d'évolution a été élaboré... !

Le livre «Sapiens » de Yuval Noah Harari qui a eu un succès international indéniable [32] est un autre exemple de relativisme. L'une des thèses de ce livre est que c'est le mythe qui fait l'homme et que la construction des mythes est indispensable à l'évolution de l'humanité. Harari pousse très loin la notion de mythe. Pour lui, le mythe concerne toute construction cognitive de la représentation d'une situation réelle ou fictive. Par exemple, un plan d'autoroute qui représente le chemin pour aller de Bordeaux à Paris et un mythe qui décrit l'existence de divinités sensées nous récompenser ou nous punir sont équivalents. Si ces deux situations ne sont pas « réelles » à proprement parler dans le sens qu'elles ne représentent pas des objets matériels précis, elles sont néanmoins très différentes. Dans la première situation (plan d'autoroute), la croyance (ou, ici, la confiance en la carte) est fondée sur des évidences qui peuvent être mises à l'épreuve (d'autres chemins existent). Dans la deuxième situation (croyance dans des divinités), l'évidence est absente. Harari évacue la notion de vérité et la façon dont elle peut être acquise d'une manière cognitive par l'expérience. II tombe dans le « anything goes » de Feyerabend qui fait de la raison un instrument comme un autre pour parvenir à la connaissance du monde. David Krakauer, du Santa Fé Institute aux États-Unis, a une toute autre vision de l'évolution cognitive ${ }^{8}$. II parle d'artifices cognitifs complémentaires (ou AC complémentaires, complementary cognitive artifacts) et d'artifices cognitifs compétitifs (AC compétitifs, competitive cognitive artifacts), ce qui semble une notion extrêmement intéressante. Une carte du monde, l'écriture et les mathématiques sont des exemples de tels artéfacts. Ces artifices peuvent modifier la structure cognitive car ils sont internalisés et n'ont plus besoin de support matériel pour fonctionner. Dans ce cas, les AC sont complémentaires. Si, en revanche, l'enlèvement des $A C$ réduit la performance, ils sont alors compétitifs.

Défense contre les conséquences du relativisme sur la notion de la nature humaine et sur la sphère sociétale

Harari étend son discours relativiste à la morale. II présente le point de vue humaniste comme une religion additionnelle, avec ses mythes et son clergé fait de philosophes rationalistes. II oublie que l'humanisme s'est construit depuis Erasme comme un mouvement indépendant des dogmes des religions, en combinant la «règle d'or » avec une vision rationnelle. La morale s'est construite sur l'altruisme, l'empathie,

\footnotetext{
${ }^{7}$ « Now the characteristic doctrine of modern irrationalists, as we have seen, are: emphasis on will as opposed to thought and feeling ; glorification of power, belief in intuition «positing » of propositions as opposed to obervational and inductive testing ». Cette phrase garde toute sa signification aujourd'hui !

${ }^{8}$ http://nautil.us/blog/will-ai-harm-us-better-to-ask-how-well-reckon-with-our-hybrid-nature
}

la sympathie, puis sur la coopération dont le point de départ est l'intentionnalité commune («shared intentionality ») qui semble être spécifiques de l'espèce humaine [33], comme le montre Michael Tomasello. Les chimpanzés et d'autres grands singes, par exemple, collaborent avec des congénères dans divers contextes pour leur bénéfice mutuel. Dans ces coalitions et alliances pour la défense du groupe, il est dans l'intérêt de tous de participer afin de vaincre l'adversaire. Chez l'homme, les jeunes enfants établissent des relations sociales entre eux au cours des premières années de vie. Ils ont aussi probablement un certain sens de leur interdépendance mutuelle. Mais bien qu'ils soient naturellement égoïstes dans de nombreuses situations, dans beaucoup d'autres, ils subordonnent leurs propres intérêts dans le but de collaborer, sympathiser et aider les autres, et partager des ressources. Ils évaluent également l'autre en termes de comportements coopératifs et commencent à aider et à partager de manière plus sélective par la suite.

D’après le primatologue Frans de Waal [34], la «théorie du vernis » postule que l'éducation et la culture donnent, seules, le cadre moral à l'homme. Initialement, l'homme est un monstre en puissance. Il doit être éduqué et rendu moral. Rien de plus faux ! L'affirmation que la morale vient de «l'intérieur » a été déjà formulée par Adam Smith [35] dans son livre «Théorie des Sentiments Moraux » et, plus récemment, par Frans de Waal [35] et Steven Pinker dans «the Blank Slate » [36].

Harari ne fait rien d'autre que de copier les théoriciens des contre-lumières, concept formulé par Isai Berlin [37], qui décrit dans son chapitre «the Counterenlightment » comment Giambattista Vico, philosophe des contre-lumières du XVIII ${ }^{e}$ siècle voyait le rôle des mythes: «Les mythes ne sont pas, comme le pensent les penseurs des lumières, de fausses descriptions de la réalité qui doivent être corrigées par l'analyse rationnelle postérieure, ni la poésie n'est pas qu'un embellissement de ce qui pourrait tout aussi bien être énoncé dans un langage simple. Les mythes et la poésie de l'antiquité incarnent une vision du monde aussi authentique que celle de la philosophie grecque, du droit romain, ou de la poésie et de la culture de notre siècle éclairé - plus tôt, éloignée de nous mais avec une propre voix authentique. Chaque culture exprime sa propre expérience collective et chacune, sur l'échelle du développement humain, a ses propres moyens d'expression authentiques ».

Les contre-lumières ont enfanté le post-modernisme dans une transformation contre-nature, dont une composante réactionnaire et traditionaliste avait pour 
but de sauver et de protéger la foi des attaques des lumières. II est curieux que ces idées aient été reprises par des philosophes issus du marxisme et transposées dans le corpus post-moderne. La notion de décolonisation de la science et surtout le mouvement «Science must fall » venant de l'Afrique du sud sont des exemples frappants des conséquences désastreuses des idées relativistes sur la sphère sociétale $^{9}[38]$. La déraison est décidemment aveugle quelle que soit la couleur politique!

La croyance, dans sa forme universaliste et absolutiste, peut se transformer en idéologie. On voit très bien les conséquences désastreuses qu'entraîne le fait de privilégier l'idéologie plutôt que la vérité. Le nazisme et le communisme soviétique et chinois sont des exemples extrêmes où le sens et la croyance étaient privilégiés par rapport à la vérité. Que cela déplaise à l'école de Francfort, ces mouvements étaient profondément irrationnels et habillés d'une rationalité de vernis ! Si la technologie est le produit de la raison, l'irrationalité peut facilement se déguiser sous le vernis de la rationalité en s'appuyant sur la technologie.

\section{Conclusion}

Comme toute activité humaine, la science n'est pas une activité purement rationnelle et il existe bel et bien des croyances et biais cognitifs qui peuvent avoir une influence sur la manière dont une recherche est conduite. Dans certains cas, ceci peut aboutir à une attitude dogmatique qui ralentit la découverte et l'innovation. Cependant, ceci n'a pas nuit d'une façon globale à son avancement.

L'esprit des lumières a été malmené par le post-modernisme, le relativisme et l'idéologie ; la notion de vérité, en particulier, a été attaquée par ces courants de pensée. Ceci peut sembler paradoxal, au vu du succès de la science dans tous les domaines. En renonçant à la vérité, la science est réduite à un jeu amusant mais sans valeur, un exercice pointu mais sans signification et à un voyage pouvant être agréable mais sans but ${ }^{10}$.

En réponse à la doxa ${ }^{11}$ de Paul Feyerabend [28] «Tout est valable d'une manière équivalente », nous souhaitons dire «tout n'est pas équivalent et valable » («Not anything goes ») et que c'est l'examen critique, utilisant la raison et l'expérience, qui en est juge. II faut donc se placer résolument dans une défense de l'esprit des lumières, comme l'a fait récemment Steven Pinker dans son livre « Enlightment now » [40]. $\diamond$

\section{SUMMARY}

\section{Science, truth and beliefs}

This article aims at discussing some aspects of the relationship between science, truth and belief. I will primarily focus on the scientific activity in the biological and medical sciences and how it relates to the notion of truth and belief and not discuss the relationship with specific

${ }^{9}$ https://www.independent.co.uk/news/long_reads/decolonise-science-time-end-imperial-era-racismbritish-empire-a8291696.html

${ }^{10}$ « By denying truth and reality, science is reduced to a pointless, if entertaining game; a mainingless, if exacting exercice; and a destinationless, if enjoyable journey » [39].

${ }^{11}$ Ensemble des opinions reçues sans discussion, comme évidentes. religions. Science has specific methodologies to obtain knowledge. Philosophers have analyzed how scientific knowledge is acquired and have tried to identify its characteristics and to establish some general rules of how knowledge through science is gained. Radical theorists have disputed the value of the scientific method despite science's indisputable successes. If science is a rational activity, it is not free from belief. Belief can have a positive and negative impact on the acquisition of scientific knowledge and the idea of human nature. These different issues will be discussed in depth in this article.. $\diamond$

\section{REMERCIEMENTS}

L'auteur remercie Jerôme Badaut (CNRS, Université Bordeaux) et Thierry Michon (INRA, Université Bordeaux) pour la relecture critique du manuscrit.

\section{LIENS D'INTÉRÊT}

L'auteur déclare n'avoir aucun lien d'intérêt concernant les données publiées dans cet article.

\section{RÉFÉRENCES}

1. Dawkins R. The mind virus. In : Dahlbom B, ed. Dennett and His Critics: Demystifying Mind : Wiley-Blackwell, 1993. 256p.

2. Durkheim $\varepsilon$. Les formes élémentaires de la vie réligieuse : CNRS editons, 1912. 300p.

3. Servetus M. CHRISTIANISMI RESTITUTIO: Restoration of Christianity, An English Translation of Christianismi Restitutio. Marian Hillar (Traduction), Christopher A. Hoffman (Traduction) : Edwin Mellen Press Ltd, 2006, 1553 : 416p.

4. Harvey W. Exercitatio anatomica de motu cordis et sanguini in animalibus. Frankfurt : Germany: Sumptibus Guiliemi Fitzeri, 1628. Forgotten Books: 262p.

5. J.S. Mill. Collected works of John Stuart Mill : VII. System of Logic : London Routeledge., 1996 (1843). 634p.

6. Popper K. Logik der Forschung: J. C. B. Mohr (Paul Siebeck), Auflage: 6. (1976) $442 \mathrm{p}$.

7. Joyce, James, "Bayes'Theorem", The Stanford Encyclopedia of Philosophy (Winter 2016 Edition), Edward N. Zalta (ed.)

8. Mayo DG. Error and the Growth of Experimental Knowledge : University of Chicago Press, 1996. 509 p.

9. Lipton P. Inference to the Best Explanation : London: Routledge, 2004. 232 p.

10. Weber M. Philosophy of Experimental Biology : Cambridge University Press $2004: 358$ p.

11. Leung DW, Cachianes G, Kuang WJ, et al. Vascular endothelial growth factor is a secreted angiogenic mitogen. Science 1989; 246 : 1306-9.

12. Ross LN. Causal selection and the pathway concept. Philosophy of Science 2018; in press.

13. Joravsky D. The Lysenko Affair : University of Chicago Press, 1970. 472 p.

14. Hübner K. Die Wahrheit des Myhos : C.H.Beck, 1985. 465 p.

15. Jung CG. The Structure and Dynamics of the Psyche: Princeton University Press; 2nd ed. edition (January 21, 1970), 608 p.

16. Tacey D. Religion as Metaphor: Beyond Literal Belief : Transaction Publishers, 2015. 286 p.

17. Kahneman D. Thinking, Fast and Slow: Penguin, 2012, 512 p.

18. Seandel M, Butler J, Lyden D, Rafii S. A catalytic role for proangiogenic marrow-derived cells in tumor neovascularization. Cancer cell 2008; 13 : 181-3.

19. Purhonen S, Palm J, Rossi D, et al. Bone marrow-derived circulating endothelial precursors do not contribute to vascular endothelium and are not needed for tumor growth. Proc Natl Acad Sci USA 2008 ; 105 : 6620-5. 


\section{RÉFÉRENCES}

20. Ayer AJ. Philosophy in the Twentieth Century. London: Weidenfeld and Nicolson, 1982. 283 p.

21. Creath, Richard, "Logical Empiricism", The Stanford Encyclopedia of Philosophy (Fall 2017 Edition), Edward N. Zalta (ed.)

22. Thornton, Stephen, "Karl Popper", The Stanford Encyclopedia of Philosophy (Summer 2017 Edition), Edward N. Zalta (ed.)

23. Musgrave A, Pigden, C. . Imre Lakatos. In : Zalta EN, ed. The Stanford Encyclopedia of Philosophy (Winter 2016 Edition)

24. Bird, Alexander, "Thomas Kuhn", The Stanford Encyclopedia of Philosophy (Fall 2013 Edition), Edward N. Zalta (ed.)

25. Preston, John, "Paul Feyerabend", The Stanford Encyclopedia of Philosophy (Winter 2016 Edition), Edward N. Zalta (ed.)

26. Kuhn TS. The Structure of Scientific Revolutions: University of Chicago Press; 3rd edition (December 15, 1996), $212 \mathrm{p}$.

27. Bikfalvi A. A Brief History of Blood and Lymphatic Vessels : Springer Verlag, 2018, $192 \mathrm{p}$.

28. P.K. Feyerabend. Against the Method: Verso, Fourth Edition (May 11, 2010) 336 pages

29. Preston J, Munevar, G., Lamb, D. The worsest ennemy of science? Essays in the memory of Paul Feyerabend: Oxford University Press, 2000, $192 \mathrm{p}$.

30. Russell B. The ancestry of fascism. In praise of idleness and other essays. London: George Allen\&Unwin 1936, $225 \mathrm{p}$.
31. Latour B. Ramsès est-il mort de la tuberculose ? La Recherche 1998; 307-.

32. Harari YN. Sapiens: A Brief History of Humankind : Vintage; દ́dition : 01 (30 avril 2015), $512 \mathrm{p}$.

33. Tomasello M. A Natural History of Human Morality : Harvard University Press, 2016, $208 p$.

34. De Waal F. Le Bonobo, Dieu et nous : Act Sud, 2013, 385 p.

35. Smith A. The Theory of Moral Sentiments.: Gutenberg Publishers, 358 p.

36. Pinker S. The Blank Slate: Penguin Books, $528 \mathrm{p}$.

37. Berlin I. The Counter-Enlightenment. Dictionary of the History of Ideas : Charles Scribner's Sons, 1973, $24 \mathrm{p}$.

38. Nordling L. How decolonization could reshape South African science. Nature 2018; $554:$ 159-62.

39. Theocharis T, Psimopoulos M. Where science has gone wrong. Nature 1987 ; $329: 4$.

40. Pinker S. Enlightenment Now: The Case for Reason, Science, Humanism, and Progress. Viking, 2018, $576 \mathrm{p}$.
TIRÉS À PART

A. Bikfalvi

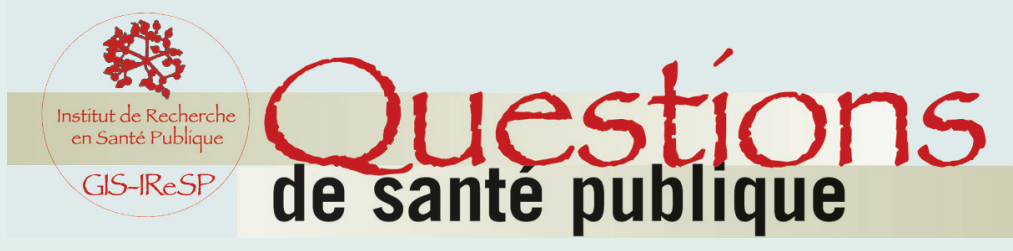

\section{In nouveau pour une meilleure visibilité des résultats bulletin de la recherche en santé publique}

es résultats de la recherche en santé publique souffrent en France d'un réel manque de visibilité. Ceci concerne aussi bien le monde académique (hors santé publique) que le grand public et les décideurs. Pour pallier ce déficit, l'IReSP a créé un bulletin à large diffusion intitulé "Questions de santé publique ", largement inspiré du bulletin mensuel d'information de l'INED " Populations et sociétés ". L'objectif éditorial est de porter à la connaissance d'un large public (enseignants, étudiants, journalistes, décideurs, milieux de la recherche, associations,

public concerné) les informations les plus récentes concernant des questions importantes de santé publique, rédigées de façon facilement lisible et compréhensible pour des non spécialistes, en garantissant que les informations publiées sont validées scientifiquement. La publication concerne des faits et non des positions. Audelà de la présentation de résultats, les qualités pédagogiques de Questions de santé publique permettent au lecteur de mieux comprendre comment sont formulées et abordées les questions de santé publique et quelles sont les limites de ces études.

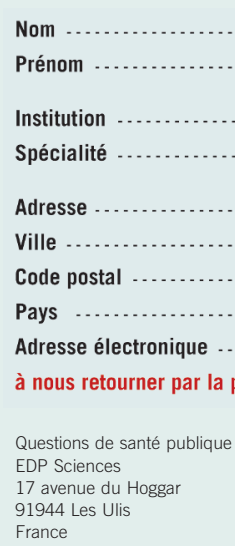

Fonction

Service 